\title{
Atomic force microscopy observation of threading dislocation density reduction in lateral epitaxial overgrowth of gallium nitride by MOCVD
}

\author{
H. Marchand ${ }^{1}$, J.P. Ibbetson ${ }^{1}$, Paul T. Fini ${ }^{1}$, Peter Kozodoy ${ }^{1}$, S. Keller ${ }^{1}$, Steven DenBaars ${ }^{1}$, J. S. \\ Speck $^{1}$ and U. K. Mishra ${ }^{1}$ \\ ${ }^{1}$ Electrical and Computer Engineering and Materials Departments, University of California, Santa Barbara,
}

(Received Sunday, February 8, 1998; accepted Tuesday, February 24, 1998)

Extended defect reduction at the surface of GaN grown by lateral epitaxial overgrowth (LEO) on large-area $\mathrm{GaN} / \mathrm{Al}_{2} \mathrm{O}_{3}$ wafers by low pressure MOCVD is demonstrated by atomic force microscopy. The overgrown GaN has a rectangular cross section with smooth (0001) and $\{11 \overline{2} 0\}$ facets. The density of mixed character threading dislocations at the surface of the LEO GaN is reduced by at least 3-4 orders of magnitude from that of bulk GaN. Dislocation-free GaN surfaces exhibit an anisotropic step structure that is attributed to the orientation dependence of the dangling bond density at the step edges.

\section{Introduction}

The lateral epitaxial overgrowth (LEO) technique consists of partially masking a substrate or "seed" layer and subsequently regrowing over the masked substrate. The potential of LEO for extended defect reduction in the heteroepitaxial growth of III-V semiconductors has been demonstrated in materials systems such as GaAs on $\mathrm{Si}$ [1], [2], InGaAs on GaAs [3], and InP on Si [4]. Recently, LEO has been utilized in the deposition of GaN, both by MOCVD [5], [6], [7] and HVPE [8], [9]. Transmission electron microscopy (TEM) studies have shown that threading dislocation (TD) reduction may occur not only by mask blocking of vertically-propagating dislocations, but also by a change in propagation direction of some dislocations at the LEO growth front [6], [9]. Such dislocations continue to propagate laterally, and so do not contribute to the TD density in the material when further growth is carried out. In one case, the resulting material (grown by HVPE) had a TD density of $\sim 6 \times 10^{7} \mathrm{~cm}^{-2}$, as compared to $\sim 1 \times 10^{10} \mathrm{~cm}^{-2}$ for the underlying $\mathrm{GaN}$ [8]. Reducing the TD density is expected to have a positive impact on the performance of GaN-based electronic and optoelectronic devices, and extended lifetime has recently been demonstrated for laser diodes grown on LEO substrates by MOVPE [10].

Atomic force microscopy (AFM) has been used in previous studies to correlate the surface morphology with the TD density of bulk GaN films grown on sap- phire by MOCVD [11], [12]. In typical high-quality $\mathrm{GaN}$ grown by MOCVD at UCSB the microstructure consists of isolated TD's of either pure edge (Burgers vector $\mathbf{b}=1 / 3\langle 11 \overline{2} 0\rangle)$, mixed $(\mathbf{b}=1 / 3\langle 11 \overline{2} 3\rangle)$ or pure screw ( $\left.\mathbf{b}=\left[\begin{array}{llll}0 & 0 & 0 & 1\end{array}\right]\right)$ character with a total density of $\sim 7 \times 10^{8} \mathrm{~cm}^{-2}$, the percentage of pure edge TD's being approximately $50 \%$ for a $2 \mu \mathrm{m}$ thick film [13]. Mixed character TD's may be distinguished by the nature of their termination. Due to their screw content with a full c-component translation, the mixed TD's terminate two $\mathrm{c} / 2$ height steps or one full $\mathrm{c}$ height step. We have previously shown that there is an excellent correlation between the mixed character TD density measured by AFM and that measured by plan view or cross-section TEM [11]. Pure edge TD's cannot terminate a step, but they occasionally give rise to small surface depressions associated with capillary phenomena. However these surface pits are not consistently observed in our material and thus the density of pure edge TD's cannot be inferred by AFM.

In this paper we use AFM to characterize the mixed character TD density reduction and the surface structure of laterally overgrown GaN grown by MOCVD. We find that the step structure of dislocation-free surface is markedly different than that of bulk GaN and thus provides useful insight into the mechanisms responsible for selective-area epitaxy and lateral epitaxial overgrowth. 


\section{Experimental}

The starting material consisted of $2 \mu \mathrm{m}$ thick GaN grown by MOCVD on 2-inch diameter sapphire wafers using a standard two-step process [14]. Samples were coated with $200 \mathrm{~nm}$ thick $\mathrm{SiO}_{2}$ using PECVD and 5-10 $\mu \mathrm{m}$ wide stripes were patterned using standard UV photolithography and wet chemical etching. Following the results of Kapolnek et al. [5], the stripes were oriented in the $\langle 1 \overline{1} 00\rangle$ direction in order to yield a large lateral growth rate. The stripe spacing was varied to give 'fill factors' (ratio of open width to pattern period) of 0.1 to 0.5 . The $\mathrm{LEO} \mathrm{GaN}$ was grown at $1080^{\circ} \mathrm{C}$ using hydrogen as the carrier gas. The sample was heated to the growth temperature under $\mathrm{NH}_{3}$. The total pressure was set to 76 Torr and the TMGa flow was $105 \mu$ moles/min. Samples were characterized by scanning electron microscopy (SEM) using a JEOL 6300F field emission microscope operating at $5 \mathrm{KeV}$ without any conductive coating applied to the sample surface. Surface topography was measured in tapping mode using a Digital Instruments Nanoscope III atomic force microscope (AFM).

\section{Results and Discussion}

Figure 1 shows a cross-section SEM micrograph of GaN overgrown from $5 \mu \mathrm{m}$ stripes with a fill factor of 0.33 after 30 minutes of growth. The sapphire substrate, the bulk GaN layer, and the overgrown $\mathrm{GaN}$ can be readily distinguished. The overgrown $\mathrm{GaN}$ is bound on top by the (0001) facet and on the edges by vertical $\{11 \overline{2} 0\}$ sidewalls. The width of the lateral overgrowth in Figure 1 is $3 \mu \mathrm{m}$ on each side, resulting in a lateral growth rate of $6 \mu \mathrm{m} / \mathrm{hr}$. We find that the morphology of the overgrown $\mathrm{GaN}$ is similar for all patterns with fill factor between 0.1 and 0.5 , but the lateral growth rate decreases with increasing fill factor. Patterns with 10 $\mu \mathrm{m}$ stripes and 0.5 fill factor are fully coalesced after 90 minutes of growth. A detailed discussion of the lateral overgrowth mechanisms will be reported elsewhere.

Figure 2 shows the surface structure of the sample shown in Figure 1b as measured by AFM. The left part of the image corresponds to GaN grown in the mask opening, whereas the $3 \mu \mathrm{m}$ wide region at the center is $\mathrm{GaN}$ overgrown over the mask. The dark stripe on the right corresponds to the $5 \mu \mathrm{m}$ deep trench at the end of the overgrown stripe and the intensity was set to black in this part of the image. Several step terminations can be seen in the GaN grown in the opening. In contrast, the laterally overgrown $\mathrm{GaN}$ is essentially free of step terminations, which indicates that the density of pure screw or mixed character dislocations reaching the surface is much lower in the overgrown GaN than in bulk GaN [11]. Transmission electron microscopy investiga- tions are under way to further assess the dislocation contents of the overgrown film and investigate the mechanisms of dislocation reduction.

Figure 2 reveals that the step structure of the overgrown $\mathrm{GaN}$ is markedly different than of the bulk GaN. In bulk GaN the steps are closely related to the surface distribution of TD's, as seen in the left part of Figure 2. In comparison the surface of the laterally overgrown $\mathrm{GaN}$ is essentially free of TD's and thus the steps tend to form well-defined patterns along crystallographic directions. The steps that are aligned with the $\langle 1 \overline{1} 00\rangle$ directions exhibit a pairing effect where the width of the terraces alternates between adjacent steps in a given direction, and between adjacent $\langle 1 \overline{1} 00\rangle$ directions for a given step (see lines A and B in Figure 2). In some cases the narrow steps seem to disappear completely in a given direction but reappear when the step orientation rotates by $60^{\circ}$, indicating that the observed pairing is not a simple step-bunching mechanism. Terraces aligned with the $\langle 2 \overline{11} 0\rangle$ directions have approximately equal widths in all directions and for adjacent steps in a given direction. They are usually seen only as a short transition between the prevalent $\langle 1 \overline{1} 00\rangle$-aligned terraces. Similar anisotropy of the step structure was observed on all samples analysed so far and is believed to be a general property of the laterally overgrown GaN under our current growth conditions. Since we are growing under conditions of high surface mobility, it is likely that the step morphology represents a local equilibrium structure.

Figure 3 shows the crystal structure of ideal Ga-terminated, un-reconstructed GaN. In this simple picture the (0001) surface of the terraces consists of gallium atoms with one dangling bond per atom. Note that the $\mathrm{GaN}$ bilayers (c/2 height) are rotated by $60^{\circ}$ for adjacent terraces, as is expected from the symmetry of the wurtzite $\mathrm{GaN}\left(6_{3}\right.$ screw axis parallel to the c axis). By minimizing the dangling bond density it can be seen that the number of dangling bonds per nitrogen atom on successive step edges in the $\langle 1 \overline{1} 00\rangle$ directions alternates between one and two. The termination type alternates between adjacent steps in a given direction, and between adjacent $\langle 1 \overline{1} 00\rangle$ directions for a given step. It is more difficult to speculate on the step edge termination in the $\langle 2 \overline{11} 0\rangle$ direction but the termination appears to be equivalent for adjacent steps in a given directions and for all $\langle 2 \overline{11} 0\rangle$ directions. Thus the anisotropy of the postulated step edge structure is consistent with our experimental observations.

Figure 4a shows a typical large-area AFM image of a $8 \mu \mathrm{m}$ wide laterally overgrown region from a 0.2 fill factor area after 90 minutes of growth. Similar surfaces free of step terminations are obtained by scanning any other area of the overgrown film over several millimeters, 
which indicates that the density of mixed character dislocations reaching the surface of the LEO GaN is in the $10^{4}-10^{5} \mathrm{~cm}^{-2}$ range. For comparison Figure $4 \mathrm{~b}$ shows the topography of a typical bulk GaN film grown at UCSB. The density of pure screw and mixed character TD's is $3.5 \times 10^{8} \mathrm{~cm}^{-2}$ as is commonly observed for bulk GaN. Thus the dislocation density at the surface of the overgrown film is reduced by at least 3-4 orders of magnitude relative to that of the bulk films.

\section{Conclusion}

Reproducible lateral overgrowth of gallium nitride on large area samples by MOCVD was achieved. Atomic force microscopy indicates a substantial reduction of the mixed character threading dislocation density by at least 3-4 orders of magnitude from typical bulk gallium nitride. It is anticipated that a similar density reduction is achieved for the pure edge dislocations. The step structure of overgrown GaN is markedly different than that of bulk $\mathrm{GaN}$ and appears to be controlled by the anisotropy of the step edge terminations rather than by the surface distribution of threading dislocations.

\section{ACKNOWLEDGMENTS}

The authors wish to acknowledge technical assistance from B. Heying. This work was supported by ONR through a contract supervised by Dr. Colin Wood. This work made use of the MRL Central Facilities supported by the NSF under award DMR9123048. HM acknowledges financial support from the Fonds FCAR (Québec), NSERC (Canada), and the Raychem Fellowship. PF ackowledges financial support from a National Defense Science and
Engineering Graduate Fellowship provided by ONR.

\section{REFERENCES}

[1] B-Y. Tsaur, R. W. McClelland, John C. C. Fan, R. P. Gale, J. P. Salerno, B. A. Vojak, C. O. Bozler, Appl. Phys. Lett. 41, 347 (1982).

[2] D. Pribat, B. Gerard, M. Dupuy, P. Legagneux, Appl. Phys. Lett. 60, 2144 (1992).

[3] K. Kato, T. Kusunoki, C. Takenaka, T. Tanahashi, K. Nakajima, J. Cryst. Growth 115,174 (1991).

[4] O. Parillaud, E. Gil-Lafon, B. Gérard, P. Etienne, D. Pribat, Appl. Phys. Lett. 68, 2654 (1996).

[5] D. Kapolnek, S. Keller, R. Vetury, R.D. Underwood, P. Kozodoy, S.P. DenBaars, U.K. Mishra, Appl. Phys. Lett. 71, 1204-1206 (1997).

[6] Tsvetanka S. Zheleva, Ok-Hyun Nam, Micheal D. Bremser, Robert F. Davis, Appl. Phys. Lett. 71, 2472-2474 (1997).

[7] O-H Nam, MD Bremser, TS Zheleva, RF Davis, Appl. Phys. Lett. 71 , 2638-2340 (1997).

[8] A Usui, H Sunakawa, A Sakai, AA Yamaguchi, Jpn. J. Appl. Phys. 36, L899 (1997).

[9] A Sakai, H Sunakawa, A Usui, Appl. Phys. Lett. 71, 2259-2261 (1997).

[10] S Nakamura, M Senoh, S Nagahama, N Iwasa, T Yamada, T Matsushita, H Kiyoku, Y Sugimoto, T Kozaki, H Umemoto, M Sano, K Chocho, Appl. Phys. Lett. 72, 211213 (1998).

[11] D. Kapolnek, X. H. Wu, B. Heying, S. Keller, B. P. Keller, U. K. Mishra, S. P. DenBaars, J. S.Speck, Appl. Phys. Lett. 67, 1541-1543 (1995).

[12] EJ Tarsa, B Heying, XH Wu, P Fini, et al., J. Appl. Phys. 82, 5472-5479 (1997).

[13] XW Wu, LM Brown, D Kapolnek, S Keller, B Keller, SP DenBaars, JS Speck, J. Appl. Phys. 80, 3228-3237 (1996).

[14] BP Keller, S Keller, D Kapolnek, WN Jiang, YF Wu, H Masui, X Wu, B Heying, JS Speck, UK Mishra, SP Denbaars, J. Electron. Mater. 24, 1707-1709 (1992). 


\section{FIGURES}

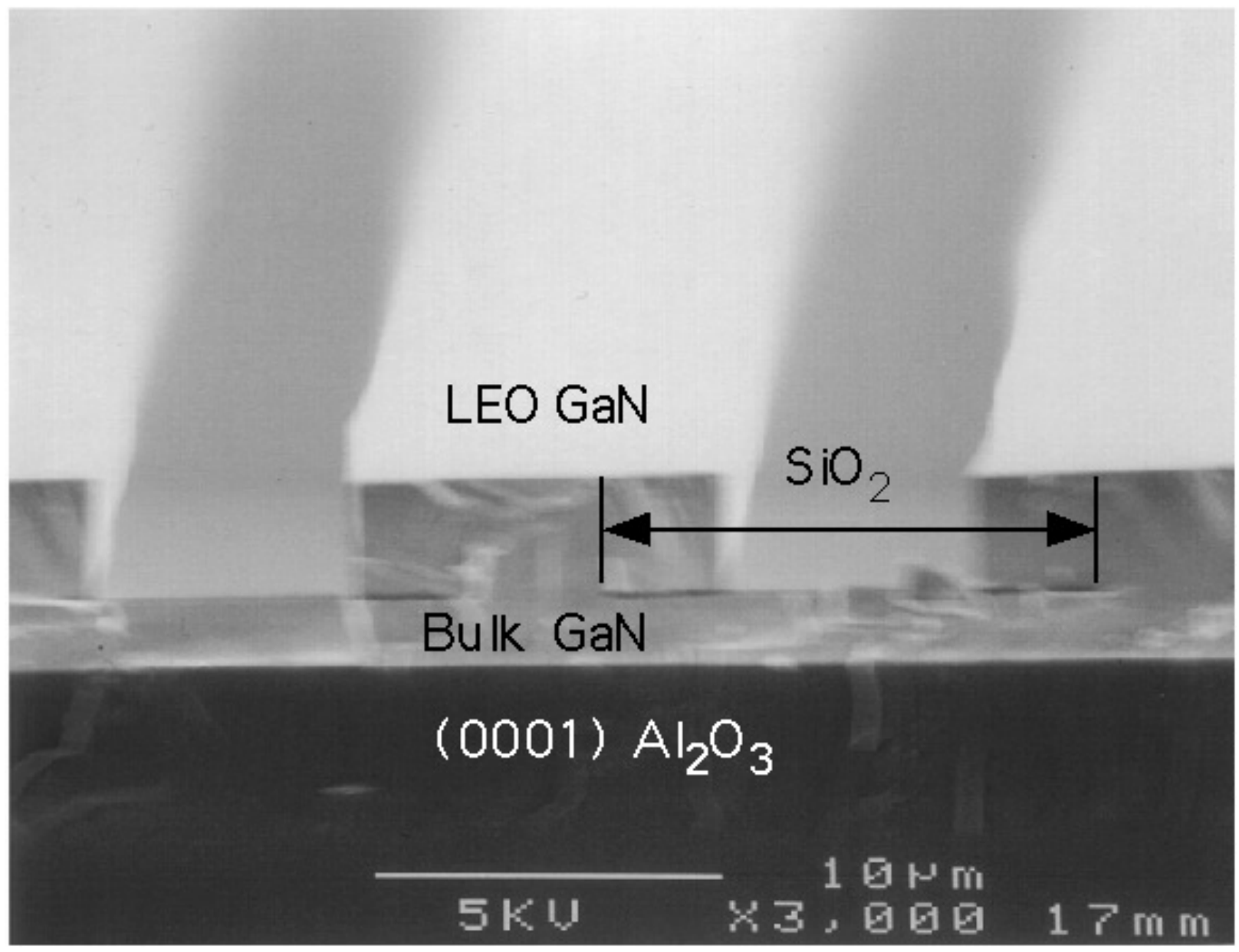

Figure 1. Cross-section SEM micrograph of laterally overgrown GaN on a $5 \mu \mathrm{m}$ stripe pattern with 0.33 fill factor after 30 minutes of growth. The stripes are aligned along $\langle 1 \overline{1} 00\rangle$. 


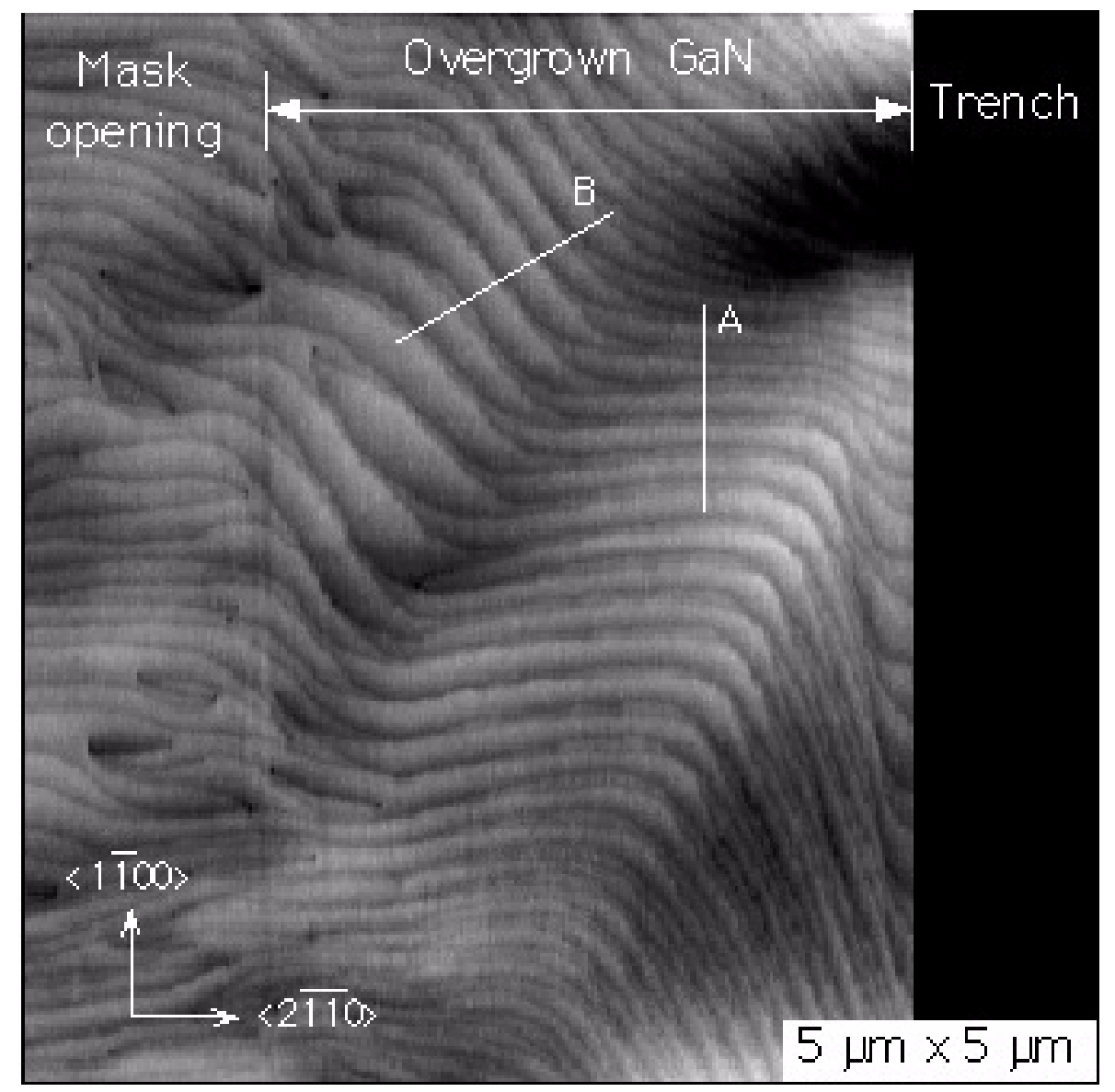

Figure 2. AFM image of the sample shown in Figure 1. The vertical range is $5 \mathrm{~nm}$.

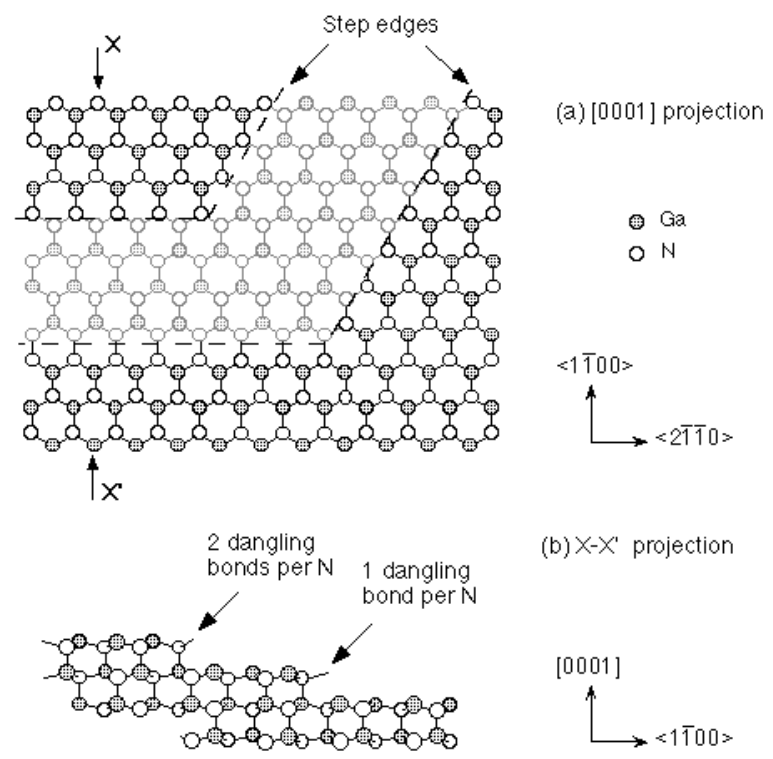

Figure 3. Schematic GaN crystal showing differences between alternating step edges in the $\langle 1 \overline{1} 00\rangle$ directions. The postulated structure of the step terminations is based on a simple model that ignores reconstruction and minimizes dangling bond densities. 


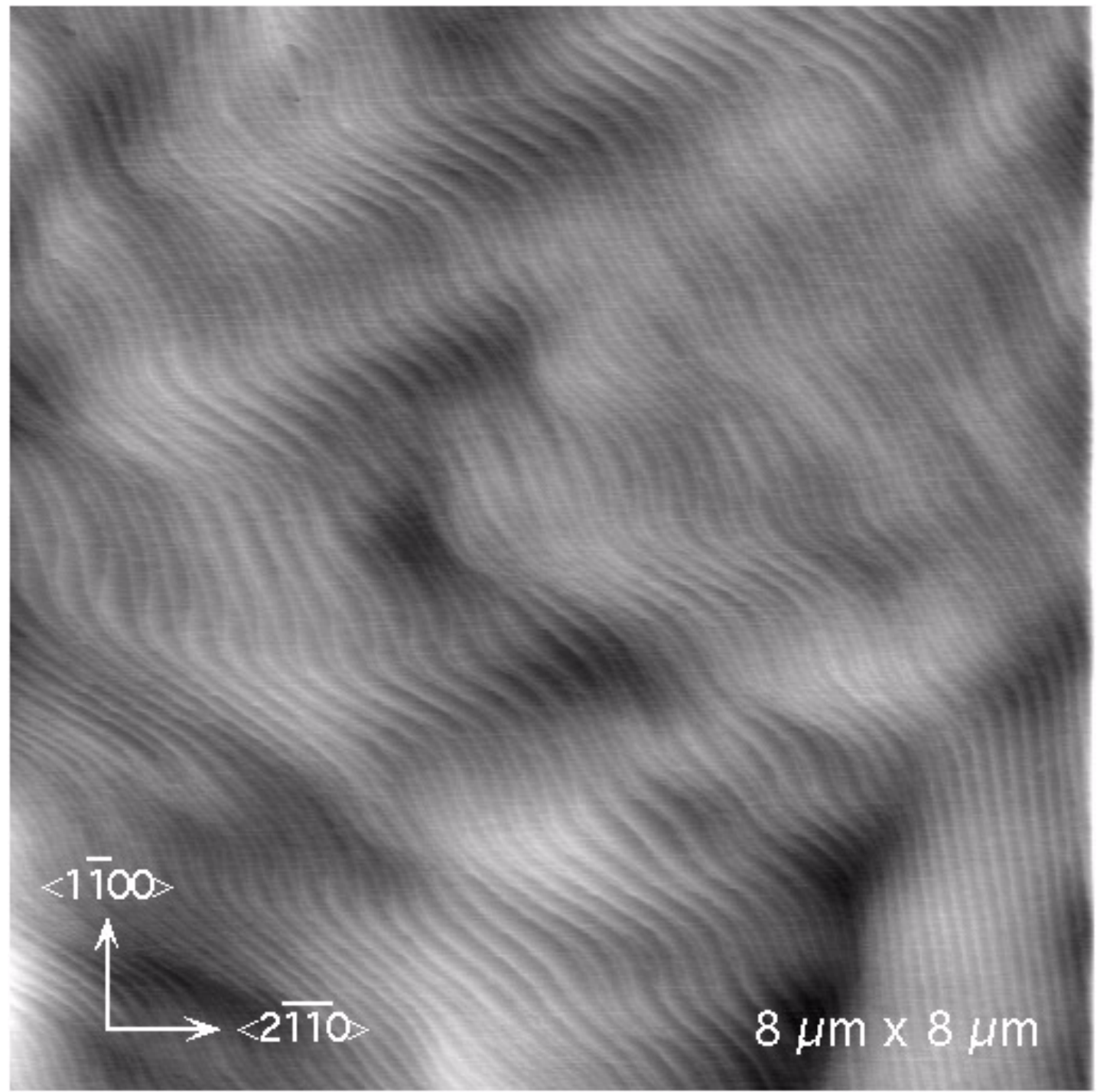

Figure 4a. AFM image of laterally overgrown GaN on a $5 \mu \mathrm{m}$ stripe pattern with 0.2 fill factor after 90 minutes of growth. The vertical range is $5 \mathrm{~nm}$. 


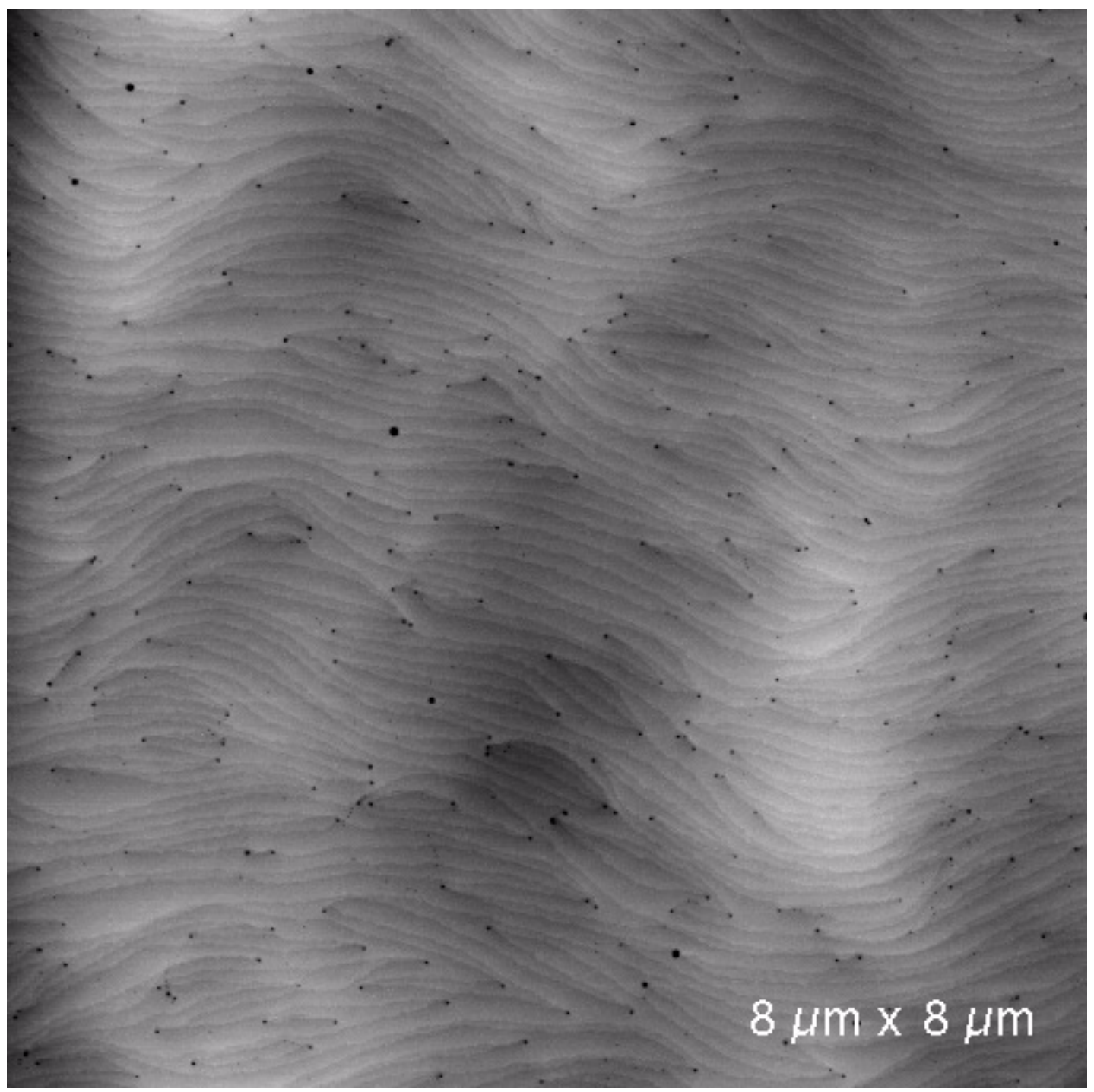

Figure 4b. AFM image of typical bulk GaN grown by MOVPE. The vertical range is $10 \mathrm{~nm}$. 